\title{
Evaluation of the Impact of Transient Disturbances on Railway Signaling Systems Using an Adapted Time-Frequency Analysis Method
}

\author{
Mohamed Raouf Kousri, Virginie Deniau, Marc Heddebaut, and Sylvie Baranowski
}

\begin{abstract}
As many other industrial environments, the railway electromagnetic environment is characterized by a large number of electromagnetic signals and disturbances. Among these, transient signals, with high energy level and wide frequency spectrum, represent an important threat to different signaling subsystems. In this paper, a new methodology dedicated to the detection and the characterization of the transient disturbances is presented. Based on a flexible and adjustable time-frequency analysis, this methodology is used to evaluate the impact of transient disturbances on a ground-to-train radio communication. A test bench was developed in order to validate the results of this evaluation.
\end{abstract}

Keywords-EMC, railways, signaling, transient disturbances, transient detection, cellular radio; Bit Error Rate

\section{INTRODUCTION}

$\mathbf{S}_{\mathrm{c}}^{\mathrm{r}}$ GNALING systems in the railway domain are continuously evolving and using new technologies. This evolution may create new forms of vulnerability to electromagnetic disturbances characterizing the railway environment. Indeed, the development of a unique interoperable railway signaling system in Europe, by creating the European Railway Traffic Management System (ERTMS) [1], introduced a new type of signaling system based notably on digital radio telecommunication. Train-to-train cellular radio using GSM-Railway (GSM-R) has become one of the main components of ERTMS. It is based on the conventional GSM standard and adapted to the railway specific applications. It allows a permanent radio connection between train and railway regulation control centers [2]. On its side, European Train Control System (ETCS) is based on the eurocab for in-train data exchange and on the eurobalise for spot communications [3]. Also part of ETCS, track circuits are used to detect the presence of a train circulating over a block [4]. Although universally

This work was supported by the french Institute of Technological Research RAILENIUM.

Mohamed Raouf Kousri is with the ITR RAILENIUM, IFSTTAR and University of Lille, France (e-mail: raouf.kousri@railenium.eu, )

Virginie Deniau and Marc Heddebaut are with the COSYS/LEOST laboratory of the French Institute of Science and Technology of Transport, Development and Networks IFSTTAR, France (e-mail: \{virginie.deniau, marc.heddebaut\} @ifsttar.fr).

Sylvie Baranowski is with the IEMN; Institue of Electronics, Microelectronics and Nanotechnology of the University of Lille, France (e-mail: sylvie.baranowski@univ-lille1.fr). based on the same principle, track circuit technologies and frequencies used can be different from one network to another.

These signaling systems are vulnerable to different types of electromagnetic disturbances, whether they are transient or permanent. The sources of these disturbances emanate from the railway infrastructure, such as the rolling stock and the power supply systems, or from the external environment, such as public cellular networks or even intentional electromagnetic interferences. The study of transient disturbances is particularly delicate. Indeed, the observed characteristics of the transient disturbances are significantly influenced by the measurement method [5]. This can produce misleading conclusions concerning their actual impact on a given system. Thus, this work is focused on the transient electromagnetic disturbances coming mainly from the sliding contact between pantograph and catenary [6], and proposes an analysis method to assess their impact on the signaling systems.

Moreover, transient disturbances are short duration signals with high power levels and wide frequency bands. These features make them able to disturb a large panel of signaling system, such as the eurobalise [7], track circuits [8], and the GSM R whose antenna is located above the train, directly exposed to the electromagnetic radiation of the sliding pantographcatenary contact [9]. Then, the analysis method proposed is also flexible to allow the assessment of their impact on different systems. However, in this paper, we will focus the analysis on the study of the transient disturbances in the GSM R frequency band (about $900 \mathrm{MHz}$ ), by adapting the analysis method to the GSM R characteristics in order to evaluate their real impact on this signaling system. Table I regroups some characteristics of the signal relevant to this study.

TABLE I

TECHNICAL CHARACTERISTICS OF GSM-R

\begin{tabular}{cccc}
\hline \hline Frequency band & $\begin{array}{c}\text { Channel } \\
\text { width }\end{array}$ & $\begin{array}{c}\text { Entry } \\
\text { filter } \\
\text { bandwidth }\end{array}$ & $\begin{array}{c}\text { Data bit } \\
\text { duration }\end{array}$ \\
\hline $\begin{array}{c}876-880 \mathrm{MHz} \text { (Uplink) } \\
921-925 \mathrm{MHZ} \text { (Downlink) }\end{array}$ & $200 \mathrm{kHz}$ & $180 \mathrm{kHz}$ & $3.7 \mu \mathrm{s}$ \\
\hline \hline
\end{tabular}

Because EMC standards in different domains, including the railway domain, were developed for analogue systems, they are not yet very well adapted to the study of the impact of transient EM disturbances on digital systems. Consequently, several research works have been carried out in order to measure and 
evaluate the impact of transient disturbances on GSM-R and GSM. These works are focused on the estimation of the bit error rate (BER) in a GSM or GSM-R communication by measuring the repetition rate of transient disturbances [10] [11], or by performing statistical characterizations of the received disturbances and correlating them with the BER [12].

Most of these studies do not take into account the inner characteristics of the system studied, such as the resolution bandwidth of its receiving filter or the time duration of the data bits. In this paper, we propose a methodology that first adapts its analysis parameters to the receiver technical characteristics in order to obtain a visualization of the transient disturbances as they appear at the input of the GSM-R receiver in terms of duration and power. Then, we proceed to the detection and the evaluation of the impact of transient disturbances on the radio communication regarding their repetition rate, power level and duration.

Section II of this paper presents the analysis methodology used based on a time-frequency analysis method. It introduces the main principle of this method and how it can be adapted to the technical characteristics of the GSM-R. Section III begins by presenting a test bench and the transient disturbance used in order to simulate and disturb a GSM-R communication. Then, it presents a method for the detection of the transient disturbances and the estimation of their effective power level at the input of the GSM-R receiver. In section IV, two methodologies allowing the evaluation of transient impact are analyzed. The first one is based on the comparison of the power level of transient disturbances to that of the GSM $\neg-\mathrm{R}$ signal. The second one employs the estimation of the real duration of transient disturbances at input of the GSM-R receiver and thus, the number of GSM-R data bits potentially corrupted. Finally, section $\mathrm{V}$ presents some perspectives and draws a conclusion.

\section{ADAPTED TIME-FREQUENCY ANALYSIS TOOL}

\section{A. Analysis Tool Presentation}

In a previous work [13], a flexible time-frequency analysis tool adapted to the analysis of different electromagnetic compatibility (EMC) issues in the railway domain has been presented. Indeed, the time-frequency analysis allows extracting simultaneously the temporal and spectral information contained in the analyzed signals [14]. For example, it is possible to obtain when each transient phenomenon appears and the frequency band that it covers. However, to exploit such an approach for EMC studies, time and frequency resolutions of the analysis have to be adapted to the temporal characteristics of the transient signals in order to ensure their detection, and, at the same time, to the technical characteristics of the system affected by these disturbances, which allows us to correctly evaluate the transient power level as received by this system. Note that, in the time frequency analyses generally used, the time and frequency resolutions are dependent on each other and cannot be correctly adapted to the EMC study [15].

The tool proposed offers the possibility to adapt the analysis time and frequency resolutions to those of each particularly studied system. The analysis is inspired from the short time Fourier transform and uses a rectangular window as the analysis window. A multi-convolution process is realized with this rectangular window in order to improve the frequency resolution. The number of convolutions is limited to 3 successive convolutions to permit conserving a sufficient time resolution [13]. Figure 1 shows the two main steps of this analysis method i.e., transforming in the frequency domain, and convolution.

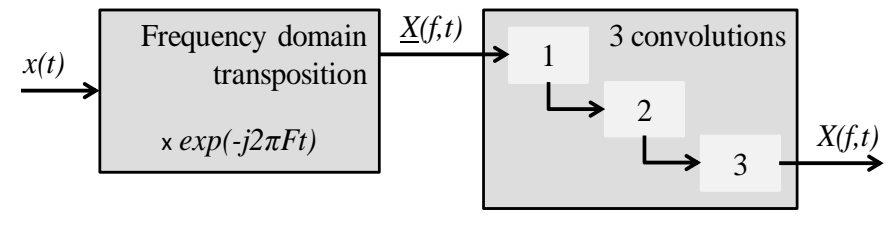

Fig. 1. Diagram of the developed analysis method.

In the first step of the method, the signal is transposed in the frequency domain by using a frequency vector $(F)$ containing the analyzed frequencies from $F_{\text {start }}$ to $F_{\text {end }}$.

In the second step, the convolution process with a rectangular window is applied to the intermediary result $(X(f, t))$, in order to obtain the time-frequency representation $(X(f, t))$. The rectangular window is selected to simplify the convolution process. Indeed, the analysis window is shifted by only 1 sample per step, thus, offering the possibility to considerably improve the time resolution. It also permits easily determining the frequency resolution according to the system studied.

\section{B. Analysis Tool Presentation}

In order to evaluate the signals and the transient disturbances as perceived by the GSM-R, the analysis has to be adapted to the frequency resolution of the entry filter of this system. In our case, it is equal to $180 \mathrm{kHz}$ at $-3 \mathrm{~dB}$ [16]. Thus, the analysis window spectrum has to be equivalent to this resolution.

The spectrum of a rectangular window which width is $\mathrm{w}$, is given by equation (1)

$$
S_{1}(f)=\operatorname{sinc}(w f)
$$

Knowing that the convolution with this window is realized 3 successive times, the resulting spectrum of this window is given by equation (2)

$$
S_{3}(f)=\operatorname{sinc}^{3}(w f)
$$

In order to obtain a desired frequency resolution which corresponds to $3 \mathrm{~dB}$ of attenuation, the width of the used window can be obtained from the following equations (3) to (6).

$$
\begin{gathered}
20 \log \left(S_{3}\left(\frac{R B W}{2}\right)\right)=20 \log \left(\operatorname{sinc}^{3}\left(w \frac{R B W}{2}\right)\right) \\
=-3 d B \\
\operatorname{sinc}^{3}\left(w \frac{R B W}{2}\right)=10^{\frac{-3}{20}}=0.7079 \\
\operatorname{sinc}\left(w \frac{R B W}{2}\right)=\sqrt[3]{0.7079}=0.8912 \\
w=\frac{\arcsin (0.8912)}{\left(\frac{R B W}{2}\right)}=\frac{0.2615}{\left(\frac{R B W}{2}\right)}
\end{gathered}
$$

Where "arcsinc" represents the inverse function of the sinc function, and $R B W$ is the frequency resolution. According to equation (6), the width $\mathrm{w}$ of the rectangular window to be applied in order to obtain a frequency resolution of $180 \mathrm{kHz}$ is $2.9 \mu \mathrm{s}$. 
The analysis is then applied to a GSM-R modulated output signal measured by an oscilloscope. Figure 2 represents a 0.2 ms time-frequency representation of this signal, obtained with an analysis window of $2.9 \mu$ s width.

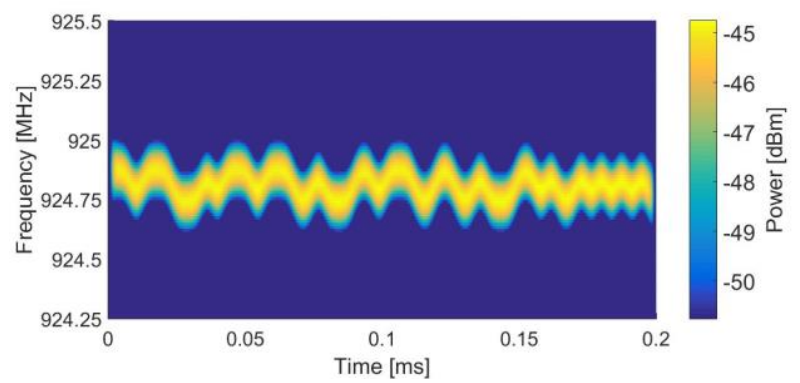

Fig. 2. Time-frequency presentation of a $0.2 \mathrm{~ms}$ GSM-R signal.

Figure 2 shows that, by adopting adequate frequency and time resolutions, this analysis tool permits us to observe in detail the evolution in the time domain of the GSM-R signal and the effect of Gaussian Minimum Shift Keying (GMSK) modulation on the frequency of the signal. In this figure, the GSM R signal frequency is $924.8 \mathrm{MHz}$. It varies in a $200 \mathrm{kHz}$ wide channel, and we can observe the $3.7 \mu$ s time duration of each data bit. These technical characteristics of the GSM-R signal are known and it is easy to adapt the analysis tool parameters to them. However, transient disturbances in the railway domain are very unpredictable and difficult to detect and characterize in order to evaluate their real impact on GSMR. Thus, the detection and analysis tool used for transient disturbances must be finely adjustable and adapted to these phenomena in the GSM R frequency band.

Measuring the quality of a digital radio link can be performed using different laboratory means. Some equipment performs an overall performance measurement by evaluating the quality of communication from one end to the other using for example a Bit Error Rate (BER) evaluation. This type of measurement, requiring a comparison between sent and received messages, can not be easily carried out during exploitation. During exploitation, online measurements, like those performed by this analysis tool, can dynamically evaluate the received signal level or the level of interference. Indeed all these measurements are tightly linked and complementary. Using the proposed analysis tool, we will show this coherence between measurements in the following sections III and IV.

\section{DETECTION AND CHARACTERIZATION OF TRANSIENT DISTURBANCES}

In order to be able to develop and evaluate the detection and characterization tool, a test bench in which a GSM-R communication is emulated is used. This test bench allows us to work in controlled experimental conditions, perfectly identifying the used GSM-R signal parameters and the superimposed transient characteristics. It also allows us to measure the real impact of these transients on the communication quality by means of BER measurement. Thus we will be able to compare these BER results with the estimated impact provided by the proposed new analysis tool, and verify the coherence between the analysis performed on the transient and the end to end BER measurement.

\section{A. Presentation of the Test Bench}

The test bench used, allowing the emulation of a disturbed GSM-R communication, is presented in Figure 3. This test bench is composed of 3 main parts:

GSM-R communication This part, denoted by 1 in Figure 3 , is composed of a cellular communication network simulator (CMU 200) and a commercial GSM-R

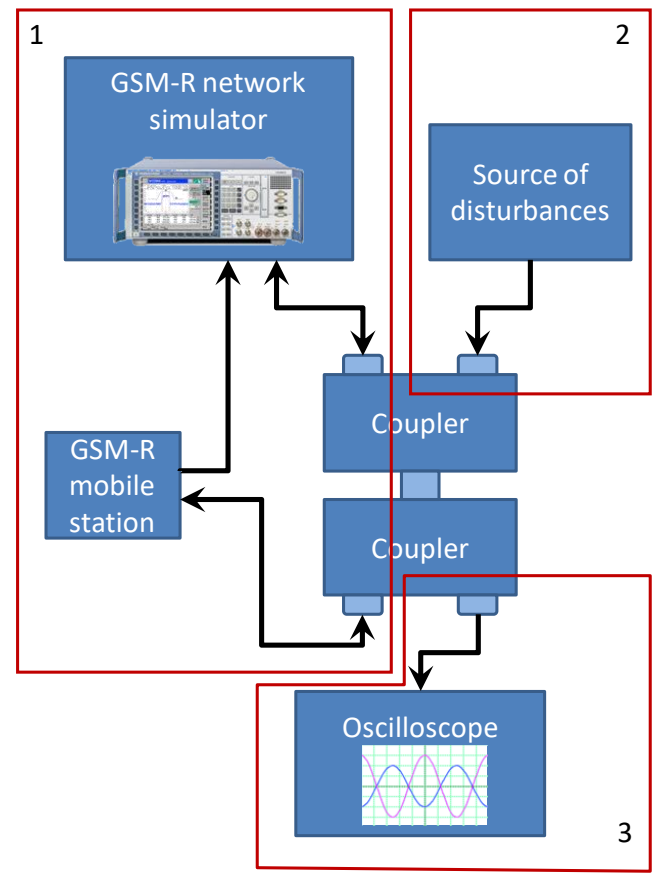

Fig. 3. Diagram of the test bench used.

are performed through coaxial lines and couplers. The frequency channel chosen for the communication is the last GSM-R channel available in the downlink band. In Europe, it corresponds to $924.8 \mathrm{MHz}$. The mobile station used in this bench has two ports. The first port allows transmitting and receiving data ( $\mathrm{Tx} / \mathrm{Rx})$ and enables communication with the CMU through the couplers and coaxial connections. The second port is a passive port, on which we find a copy of the signal at the first port with a reduction in power. This port is connected directly to the CMU and realizes the "Loop back" function in the test bench. This function permits us to retrieve the data exchanged without passing through the couplers, so avoiding the disruption on this path. This allows the CMU to evaluate the channel quality by comparing the data exchanged over the channel to those received through the "Loop back", thus determining the bit error rate (BER) of the transmitted data.

Transient disturbances generation The second part, denoted by 2 in Figure 3, consists of a source of transient disturbances, which, in this test bench, is an arbitrary waveform generator (AWG). This equipment is then able to generate the transients with an adjustable amplitude and occurrence rate.

Acquisition The last part of the bench, denoted 3 in Figure 3, is composed of a digital oscilloscope connected to the output port of the second coupler. The oscilloscope acquires the GSM$\mathrm{R}$ signals exchanged and stores them in $0.2 \mathrm{~ms}$ duration files sampled at $2.5 \mathrm{GSa} / \mathrm{s}$, providing recordings of $5 \times 10^{5}$ samples each. 


\section{B. Transient Disturbances Used}

The transient disturbances used in this study are synthesized signals whose characteristics are based on in situ measurements in the GSM-R frequency band presented in [17]. They last $20 \mathrm{~ns}$ and are created using a double exponential waveform modulated by a $923 \mathrm{MHz}$ frequency, which is the center frequency of the European GSM-R allocated band. This signal is loaded in the AWG to be injected into the communication channel with the GSM-R signals. The occurrence rate of the transient disturbance is adjusted to $50 \mu \mathrm{s}$ between each appearance. An example of a signal generated with this configuration and measured at the output port of the second coupler is represented figure 4.

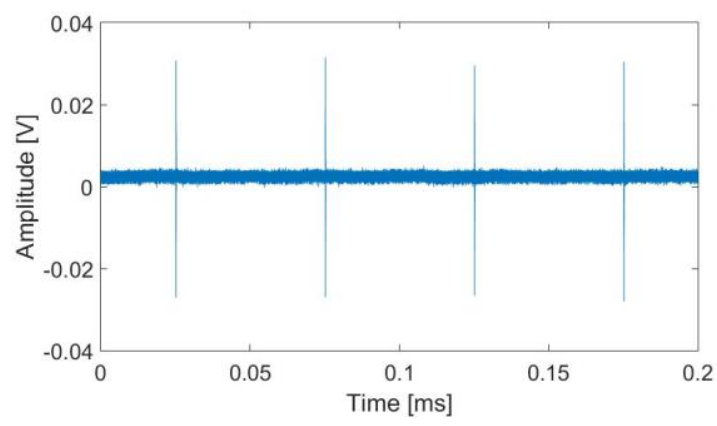

Fig. 4. Time representation of the GSM-R signal with transient disturbances every $50 \mu \mathrm{s}$.

We notice that the transient signals are significantly superior to the communication signal. However, this observation in time domain does not permit us deducing the real impact on the communication quality.

The aim of this study is to automatically detect transient phenomena, determine their power level and time duration, such as perceived by the GSM-R receiver. Then, we compare them to the GSM-R signals power and to the GSM-R bit time duration and we check the relevance of the result by comparison with the measured BER.

\section{Transient Detection Method}

\section{Analysis window width}

In order to optimize the detection of the transient disturbances, the analysis window width used has to be adjusted to the duration of these disturbances. In the GSM-R frequency band, this duration is approximately $20 \mathrm{~ns}$ [17]. However, the use of this very short window width induces a poor frequency resolution and the spreading of the signal power over a wide frequency band. Figure 5 is the time-frequency representation of the signal presented previously in figure 4 . This result is obtained using a $20 \mathrm{~ns}$ rectangular window width.

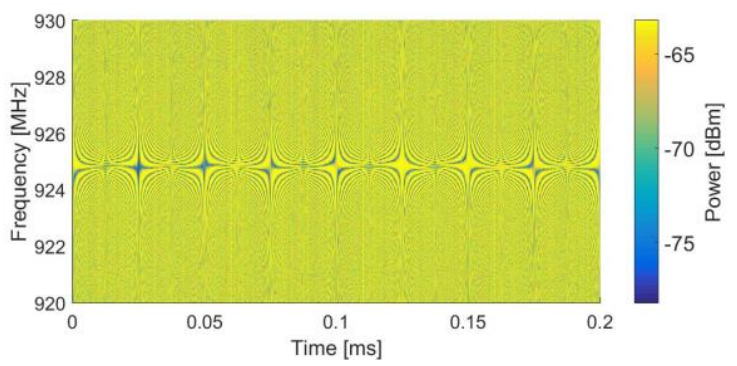

Fig. 5. Time-frequency representation of the analyzed signal with an analysis window width of $20 \mathrm{~ns}$.
Figure 5 shows that the entire frequency band analyzed seems to be covered by the GSM-R signal. Indeed, the large resolution bandwidth of the analysis window used $(26.15 \mathrm{MHz})$ due to its short duration (20 ns) spreads the energy of the GSM-R signal over a large frequency band. In this case, the transient disturbances become difficult to detect. Note that, without any permanent signal in the analyzed band, transient disturbances would be easily detectable.

Moreover, the use of a large analysis window $(2.9 \mu \mathrm{s})$, adapted to the GSM-R entry filter resolution bandwidth (180 $\mathrm{kHz}$ ), induces a limitation of the time resolution, making the transient disturbances difficult to detect, as shown in figure 6 .

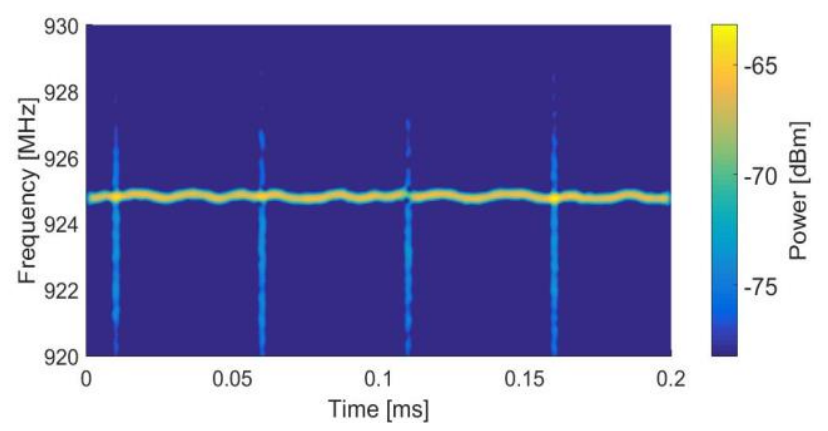

Fig. 6. Time-frequency representation of the signal analyzed with an analysis window width of $2.9 \mu \mathrm{s}$.

Indeed, the use of a large window compared to the duration of a transient phenomenon spreads the power of this transient across the full width of the window used. It results in an apparent decrease in its calculated power which affects the detection. The relationship between the window width used and the transient calculated power will be described later in this paper.

Using these initial analyzing tool settings, we conclude that the detection and characterization of transient disturbances is imperfect. Then, an intermediary analysis window width is required to analyze a complex signal comprising both transient and permanent components. This new window width should permit us to increase the transient disturbance power, and, at the same time, to avoid spreading the permanent signal power over the analyzed frequency band. Thus, transient disturbances become more easily detectable, and we can correctly estimate their power, without any influence of the permanent signals, by measuring them in an empty frequency channel.

We know that transient disturbances are large spectrum phenomena. Therefore, initially, we assume that they are equally distributed over the $4 \mathrm{MHz}$ allocated band. Consequently, measuring the transient disturbance power in a close, unoccupied frequency channel is equivalent to measuring it in the GSM-R channel used (924.8 MHz). Thus, we can deduce a satisfying estimation of their impacts on the GSM-R system.

The choice of the intermediary analysis window must be a compromise allowing the selection of an unoccupied frequency channel to detect the transient disturbances. Until now, this choice has depended on the operator's skill and is based on the spectrum observation. However, it should be feasible to develop an automatic method able to analyze and choose a suitable channel for the detection, because in practice, the GSM-R frequency band is less occupied than that of the public 
cellular phone bands. This method will be developed in upcoming works.

Figure 7 shows the time-frequency representation of the signal obtained with an analysis window of $0.33 \mu$ s width.

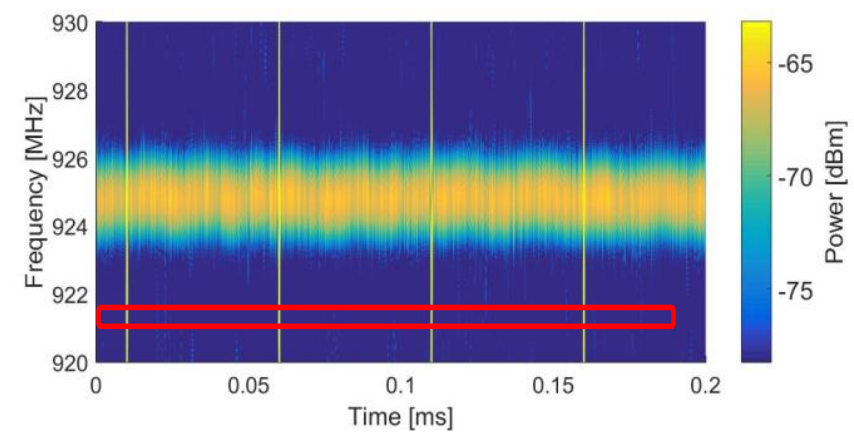

Fig. 7. Time-frequency representation of the analyzed signal with an analysis window width of $0.33 \mu \mathrm{s}$

TABLE II

Evolution of THE TRANSIENT DistURBANCE POWER With THE ANALYSIS WINDOW WIDTH.

\begin{tabular}{ccccccc}
\hline \hline Width [ns] & 5 & 10 & 20 & 40 & 80 & 160 \\
\hline Power [dBm] & -12.8 & -19.3 & -25.6 & -30.6 & -37.3 & -44.4 \\
\hline \hline
\end{tabular}

In figure 7, there are different non occupied channels offering the possibility to correctly detect and measure the power of the transient disturbances, which is also verified in situ, because generally only one channel is employed by GSM-R cell. Therefore, we select the $921 \mathrm{MHz}$ free channel to perform the process. Thus, the analysis is still realized in the GSM-R allocated frequency band.

Effect of the window width on the computed power and determination of a correction factor

To highlight the effect of the analysis window width on the determined transient power value measurement, 3 different window widths are used i.e., $5 \mathrm{~ns}, 10 \mathrm{~ns}$ and $20 \mathrm{~ns}$. The short durations of these windows imply that the frequency resolution is very large $(200 \mathrm{MHz}$ in the case of the $5 \mathrm{~ns}$ window). Measurement should thus be carried out at at least $200 \mathrm{MHz}$ margin from the GSM-R frequency band, i.e. about $700 \mathrm{MHz}$. To compare the transient disturbance power obtained with these 3 widths, Figure 8 provides a representation in "zero span" of the $700 \mathrm{MHz}$ channel obtained from the time-frequency analysis of the analyzed signal. This "zero span" representation allows seeing the evolution of this channel through time. At this frequency, the calculated power is not affected by the GSM-R permanent signal despite the poor frequency resolution induced by the short durations of the windows and this permits us to only observe the impact of the window width on the obtained transient signal power level.

Figure 8 shows that the calculated power of the transient, circled in blue, decreases as the size of the analysis window increases. Table II illustrates the evolution of the value of this power according to the size of the window.

According to Table II, each time the analysis window doubles in width, the transient power is reduced by about $6 \mathrm{~dB}$. This is consistent with our transient interference characteristics since we are considering broadband phenomena, whose power is uniformly distributed over the frequency band of interest.
Thus, we can deduce a relationship between the different determined powers of the transient disturbance and each window width.

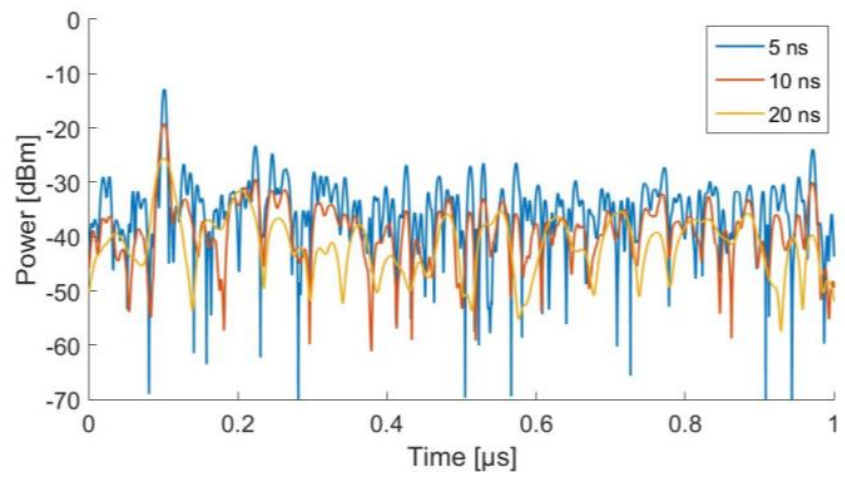

Fig. 8. Zero span representation of the $700 \mathrm{MHz}$ frequency channel obtained from time-frequency analysis of the transient disturbances used and by applying 3 different analysis window widths.

Assuming that the analysis is realized with two different sizes of window, $w_{1}$ and $w_{2}$, and the ratio between the two sizes is $r=w_{1} / w_{2}$, the respective powers $P_{l}$ and $P_{2}$, in $\mathrm{dBm}$, obtained with these two windows are given by equations (7) and (8).

$$
P_{1}=20 \log \left(x_{1}\right)+13
$$

$$
P_{2}=20 \log \left(x_{2}\right)+13
$$

$x_{1}$ and $x_{2}$ are the analysis results obtained respectively with the window widths $w_{1}$ and $w_{2}$. As the analyzed signals are measured across a $50 \Omega$ resistance, the value 13 permits the conversion from $\mathrm{dBV}$ into $\mathrm{dBm}$.

The relationship between $x_{1}$ and $x_{2}$ is given by equation (9)

$$
x_{2}=x_{1} r
$$

$P_{2}$ can therefore be deduced from $P_{1}$ by using equation (10)

$$
P_{2}=P_{1}+20 \log (r)
$$

Therefore, $20 \log (r)$ can be considered as a correction factor. Knowing $P_{1}$, it permits to determine the value of $P_{2}$, which in our case, corresponds to the power of transient disturbances collected by a GSM-R receiver.

\section{EVALUATION OF TRANSIENT DisTURBANCES IMPACT ON GSM-R}

\section{A. Comparison of the Transient Power with the GSM-R Signal Power}

The presented process aims ultimately to be a substitute for measuring the actual BER in presence of interference, not accessible during GSM-R exploitation.

As mentioned previously, the transient disturbances are first detected by using the intermediary window width of $0.33 \mu$ s. Their power is determined on the $921 \mathrm{MHz}$ frequency channel. In order to evaluate their impact on GSM-R communication, the power determined is compared to the power level of the GSM-R communication signal.

In figure 9, both GSM-R signal (924.8 MHz) and transients at $921 \mathrm{MHz}$ are presented together. The blue curve represents the GSM-R signal power obtained with a $2.9 \mu$ s window; the black curve corresponds to the $921 \mathrm{MHz}$ channel power 
obtained with the $0.33 \mu$ s window, and finally, the red curve is the mean value of the GSM-R signal power. In the first step, the amplitude of these transient disturbances is chosen in a way that the measured BER is equal to $0 \%$. In other words, these disturbances have no impact on the GSM-R communication quality.

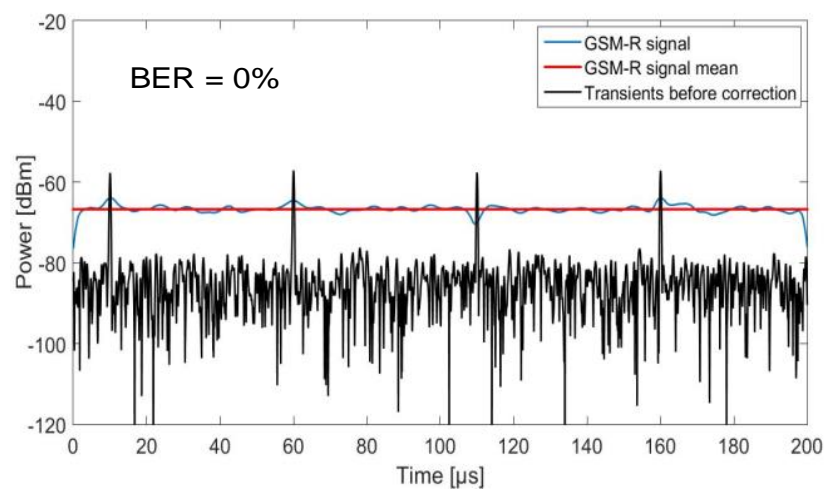

Fig. 9. Comparison between the GSM-R signal power level and the transient disturbance power level before correction. Case of no disturbance impact.

As shown in figure 9, before applying the correction factor, the power of the transient disturbances detected is above the power of the GSM-R signal, which does not match with the real level, as verified by the satisfactory measured BER.

Figure 10 compares the powers of the same channels after adding the correction factor, which is only applied to the transient disturbances. The power level of these disturbances is obtained by applying the equation (11)

$$
P_{2}(\mathrm{dBm})=P_{1}+20 \log \left(\frac{0.33 \mu \mathrm{s}}{2.9 \mu \mathrm{s}}\right)
$$

P1 corresponds to the power of transient disturbances presented in figure 9 and determined by using a $0.33 \mu$ s window width. The correction factor is added to the instants of occurrence of these disturbances, which explains that only their power level decreases in figure 10.

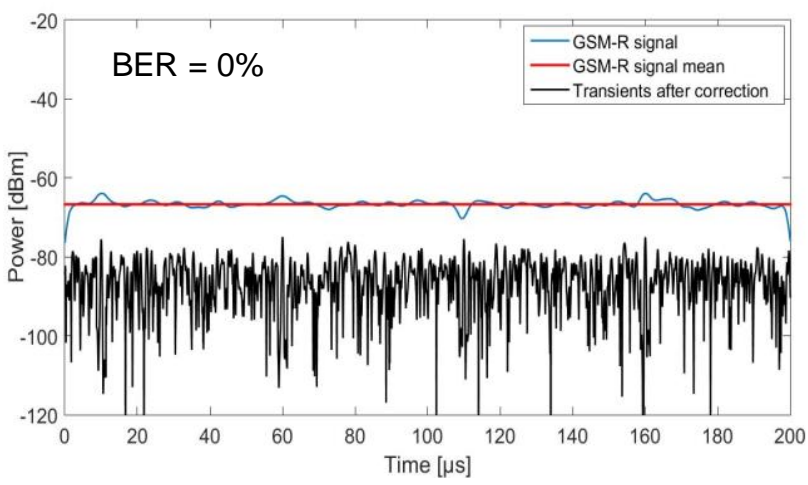

Fig. 10. Comparison between the GSM-R signal power level and the transient disturbance power level after correction. Case of no disturbance impact.

Figure 10 shows that, after the correction, the transient disturbance power is comfortably below the GSM-R signal. This result is consistent with the measured BER of $0 \%$. Indeed, these disturbances do not have any impact on the signaling system and the communication quality is not affected.
A second case is presented where the amplitude of the transient disturbances is higher by $13 \mathrm{~dB}$ than in the first case. The measured BER is now equal to $1 \%$, which means that these transients have a significant impact on the GSM-R signal. Indeed, for safety reasons in the railway domain, the BER value of GSM-R communication must not exceed $1.6 \%$ during train operation [18].

Figure 11 shows a comparison between the GSM-R signal and the transients measured at $921 \mathrm{MHz}$, before applying the correction factor.

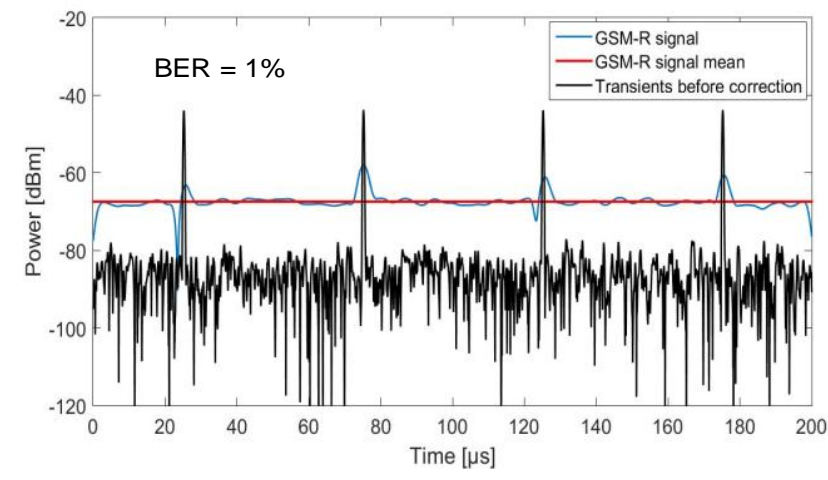

Fig. 11. Comparison between the GSM-R signal power level and the transient disturbance power level before correction. Case of high disturbance impact.

In figure 12, the correction factor is applied to the transient disturbances, as described previously. Also, the correction factor remains the same since we are using the same window widths.

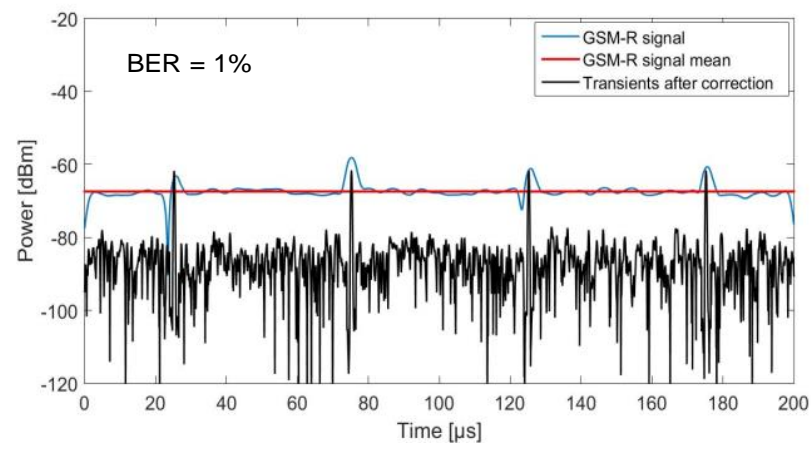

Fig. 12. Comparison between the GSM-R signal power level and the transient disturbance power level after correction. Case of high disturbance impact.

In figure 12, and after applying the correction factor, the power of the transient disturbances still exceeds the level of the GSM-R signal power. This result is concordant with the measured BER of $1 \%$ since it indicates that the detected transient disturbances impact the communication quality. Indeed, previous research works indicate that transient disturbances directly affect the quality of a GSM communication once their power equals or exceeds the power level of this communication [19].

Thus, the detection method developed permits us to separate the transient disturbances that have an impact on the signaling system from those that do not. This is ensured by comparing the real power level of each transient with the useful signal power level. Thus, this detection method is more efficient than using a trigger and choosing an arbitrary threshold to detect transient. 


\section{B. Transient Disturbance Characteristics in the Time Domain}

Beside their power level, the time domain characteristics of the transient disturbances are very important in the study of their impact on the GSM-R. Indeed, this system is a digital communication system that uses digital data frames in which each data bit lasts $3.7 \mu$ s. Therefore, the duration of electromagnetic disturbances, whether they are shorter or longer than $3.7 \mu \mathrm{s}$, has an impact on the number of disturbed data bits, and thus on the quality of the digital communication.

Previous works have studied the time domain characteristics of the transient disturbances in the GSM-R frequency band by the determination of their duration, rise and fall time [17]. However, these studies do not take into account the characteristics of the GSM-R systems. Indeed, the entry filter of the GSM-R introduces a time delay on the received disturbances, which enlarges their duration and thus raises their impact on the digital communication. Thus, a transient signal lasting some ns can be transformed into a transient signal lasting some $\mu$ s due to the time constant of the receiver filters.

We have already seen that the analysis tool developed can be adapted to the GSM-R technical characteristics by adjusting its analysis window width to $2.9 \mu \mathrm{s}$, which corresponds to its resolution frequency bandwidth $(180 \mathrm{kHz})$. However, the transient disturbance detection is ensured by the use of a narrower analysis window, and thus gives a wrong estimation of these phenomena. As in the case of the power level estimation, a correction factor can be used in order to obtain the effective duration of the detected transient at the input of the GSM-R receiver. We are interested in the duration in which the transient disturbance power is higher than the mean value of the GSM-R signal power. Indeed, it is the time in which these disturbances have an impact on the GSM-R. The corrected duration, $\mathrm{Dc}$, is given by equation 12 .

$$
D_{c}=D_{i} \times \frac{w_{2}}{w_{1}}
$$

$D_{i}$ is the duration obtained with the intermediary window width employed for the detection, $w_{2}$ is the width of the large analysis window, equal to $2.9 \mu \mathrm{s}$, which corresponds to the GSM-R receiver filter, and $w_{l}$ is the width of the intermediary window, which is equal to $0.33 \mu \mathrm{s}$.

Figure 13 shows two representations of the transient disturbances. They are obtained using the intermediary (black curve) and the large (green curve) analysis windows respectively. The durations in which these transients exceed the mean value of the GSM-R signal power are also given.

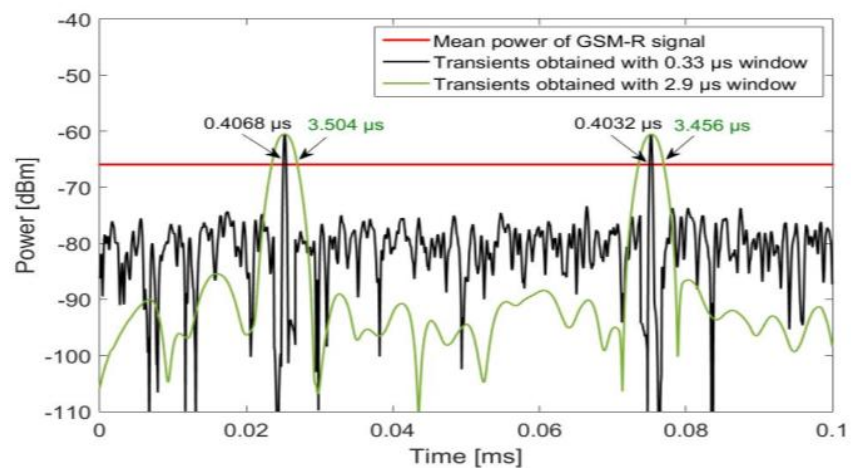

Fig. 1. Representations of the transient disturbances obtained by the intermediary window (black) and the large window (green).
As we can see in figure 13 , the duration $D_{i}$ obtained with the intermediary window is about $0.4 \mu \mathrm{s}$, while the real duration, $D_{r}$, obtained with the large window is about $3.5 \mu$ s. The duration $D_{i}$ is then corrected regarding the GSM-R entry filter characteristics. The corrected value of this duration is obtained from equation (13)

$$
D_{c}=D_{i} \times \frac{2.9 \mu s}{0.33 \mu s}
$$

The results of this correction concerning the transients of figure 13 are presented in table III.

TABLE III

INTERMEDIARY, CORRECTED AND REAL DURATIONS OF THE TRANSIENT DISTURBANCES.

\begin{tabular}{cccc}
\hline \multicolumn{4}{c}{ TRANSIENT DISTURBANCES. } \\
\hline \hline First transient & $0.407 \mu \mathrm{s}$ & $3.575 \mu \mathrm{s}$ & $3.504 \mu \mathrm{s}$ \\
\hline Second Transient & $0.403 \mu \mathrm{s}$ & $3.543 \mu \mathrm{s}$ & $3.456 \mu \mathrm{s}$ \\
\hline \hline
\end{tabular}

Table III shows that the corrected durations obtained from the intermediary are considerably close to the values of the real duration obtained with the large window. This permits us to conclude that the method used that detect and estimate the real duration of the transient disturbances is an efficient method which will allow us to use it in real time applications thanks to its reliability and its fast calculation times.

Table III shows also that the duration of the transient disturbances passes from $20 \mathrm{~ns}$ to about $3.5 \mu \mathrm{s}$, which is equivalent to the duration of a GSM-R data bit. They can be longer if their power is higher. This means that these transient disturbances are able to impact 1 or 2 data bits at once, which will have a significant impact on the BER and thus on the GSM$\mathrm{R}$ communication quality.

With this in mind, we conclude that not only the power and the frequency of occurrence of transient disturbances must be taken into account in the study of their impact on the GSM-R, but also their duration. During our measurements, we noticed that the more powerful transient disturbances, the longer their durations. This implies the need for further research works to find a link between these 2 parameters and the BER of a digital communication system.

\section{CONCLUSION}

Detection and characterization of transient electromagnetic disturbances are very important in order to evaluate their impact on systems. To effectively evaluate their impacts, these disturbances have to be considered as received by the disturbed system entry filter, and not only as emitted by the sources. Therefore, in this paper, we propose a methodology dedicated to the detection and the characterization of transient disturbances adaptable to the technical characteristics of a studied signaling system. We have selected the case of GSM-R signals. This methodology is based on an adaptable time frequency analysis method. The methodology starts by adapting the analysis window width in order to be able to detect the transient disturbances in the GSM-R frequency band. Then, a correction factor is applied to each detected transient in order to obtain its power and duration as received by a real GSM-R entry filter. The power is then compared to the GSM-R signals 
and the duration to the data bit duration, which allows evaluating the real impact of the transient disturbances on this system.

In this paper, the methodology proposed is applied to EMC issues that concern railway signaling systems. However, the flexibility of this methodology and of the analysis tool that it is based on, allows the enlargement of its application scope to other domains, such us the production and transfer of electric energy, medical equipments or cyber security.

\section{REFERENCES}

[1] Intenational Union of Railways (IUC), "ERTMS," [Online]. Available: http://uic.org/ertms.

[2] M. Palumbo, "The ERTMS/ETCS signalling system," [Online]. http://www.railwaysignalling.eu/wp-content/uploads/2014/08/ERTMS_ ETCS_signalling_system_MaurizioPalumbo1.pdf. August 27, 2014.

[3] UNISIG, "FFFIS for Eurobalise, SUBSET-036, Issue: 3.0.0," February 24, 2012.

[4] M. Laughton and D. Warne, "Railways," in Electrical Engineer's Reference Book, 16 ed., Newnes, 2002, pp. 14-16.

[5] J. Barros, M. Apraiz and R. I. Diego, "Review of signal processing techniques for detectionof transient disturbances in voltage supply systems," Proc. IEEE International Instrumentation and Measurement Technogy Conference., pp. 450-455, May 2013.

[6] S. Midya, D. Bormann, Z. Mazloom, T. Shüttle and R. Thottappillil, "Conducted and Radiated Emission from Pantograph Arcing in AC Traction System, " IEEE Power \& Energy Society General Meeting, Calgary, pp 1-8, July 2009.

[7] I. Adin, J. Mendizabal, S. Arrizabalaga, U. Alvarado, G. Solas and J. Rodriguez, "Rolling stock emission testing methodology assessment for Balise Transmission Module system interoperability," Measurement, vol. 77, pp. 124-131, January 2016.

[8] European Railway Electromagnetic Compatibility (EUREMCO), "D2.2 Transients Characteristic Parameters," November 30, 2012.
[9] A. Mariscotti, A. Marrese and N. Pasquino, "Time and frequency characterization of radiated disturbance in telecommunication bands due to pantograph arcing," Measurement, vol. 46, no. 10, pp. 4342-4352, December 2013.

[10] G. Boschetti, A. Mariscotti and V. Deniau, "Assessment of the GSM-R susceptibility to repetitive transient disturbance," Measurement, vol. 45, no. 9, pp. 2226-2236, November 2012.

[11] R. Adriano, N. Ben Slimen, V. Deniau, M. Berbineau and P. Massy, "Prediction of the BER on the GSM-R communications provided by the EM transient disturbances in the railway environment," International Symposium on Electromagnetic Compatibility - EMC Europe 2008, pp. $1-5$, October 2008.

[12] M. Pous and M. A. Azpura, "Measurement and Evaluation Techniques to Estimate the Degradation Produced by the Radiated Transients Interference to the GSM System," IEEE Transaction on Electromagnetic Compatibility, vol. 57, no. 6, pp. 1382 - 1390, December 2015.

[13] M. R. Kousri, V. Deniau, M. Heddebaut, S. Baranowski and J. Rioult, "Time-Frequency Processing Adapted for the Different Electromagnetic Compatibility Issues in the Railway Domain," in IEEE International Symposium on Electromagnetic Compatibility (EMC), Dresden, August 2015 .

[14] S. Qian and D. Chen, "Joint time-frequency analysis," Signal Processing Magazine, IEEE, vol. 16, no. 2, pp. 52-67, March 1999.

[15] W. Pan and D. Pommerenke, "Emi failure analysis techniques: II. joint time-frequency analysis," IEEE EMC Society Newsletter, vol. 226, pp. $31-34,2010$.

[16] Maxim Integrated, "Introduction to GSM and GSM Mobile RF Transceiver Derivation," [Online]. http://pdfserv.maximintegrated.com/en/an/AN2157.pdf. July 04, 2003.

[17] V. Deniau, N. Ben Slimen, S. Baranowski, H. Ouaddi, J. Rioult and N. Dubalen, "Characterisation of the em disturbances affecting the safety of the railway communication systems," Eur. Phys. J. Appl. Phys., vol. 43, no. 2, pp. 225-230, August 2008.

[18] International Telecommunication Union, "ITU-T K.48: EMC requirements for telecommunication equipment - Product family Recommendation," September 2006.

[19] S. Dudoyer, V. Deniau, S. Ambellouis, M. Heddebaut and A. Mariscotti, "Classification of Transient EM Noises Depending on their Effect on the Quality of GSM-R Reception," IEEE Transactions on Electromagnetic Compatibility, vol. 55, no. 5, pp. 867 - 874, October 2013. 